\title{
Application of 3D EBSD to a Two-Phase, Ultra-Fine Grain Titanium Alloy
}

\author{
A. Buxbaum ${ }^{1}$, S.D. Sitzman $^{2}$, and A. Deal ${ }^{3}$ \\ ${ }^{1}$ FEI Company, Hillsboro, OR 97124 \\ ${ }^{2}$ Oxford Instruments America, Concord, MA 01742 \\ ${ }^{3}$ GE Global Research, Niskayuna, NY 12309
}

Electron BackScatter Diffraction (EBSD) is a powerful characterization technique that continues to gain widespread acceptance in the materials science and geological communities. Variants of EBSD are now extending it's experimental capabilities in interesting new ways, e.g. for in-situ high temperature transformation and grain growth monitoring [1], and for 3D microstructural characterization [2]. The latter, 3D EBSD, has progressed significantly in flexibility and ease of use as FIB-SEM (DualBeam ${ }^{\mathrm{TM}}$ ) and EBSD hardware and software continues to develop. Yet, considerable care is still required to obtain high quality data and reconstruct accurate $3 \mathrm{D}$ microstructural representations, especially in determining true grain size \& shape and the associated variety of useful analyses, such as 3D grain boundary plane characterization. This necessity is particularly acute for fine grain materials.

To better understand the influence of key factors in 3-D EBSD acquisition and processing on fine grain materials, we examined a two-phase titanium alloy with a sub-micron average grain size in both the alpha and beta phases, processed by Multi-Axis Forging (MAF) [3]. A backscattered electron image is presented in FIG 1. This material presented significant milling and diffraction challenges. Regarding milling, titanium has a relatively high melting point and theoretically produces a low bulk sputter yield of 13.28 atoms/ion at glancing ion beam incidence [4]. Regarding diffraction, although the alpha phase generally produces reasonable EBSD patterns, the softer beta phase is much more susceptible to mechanical damage, reducing the quality of the beta patterns. Despite these difficulties, 3D data acquisition was accomplished on an FEI Quanta 3D FEG DualBeam $^{\mathrm{TM}}$ equipped with EBS3 and an Oxford Instruments HKL EBSD system using the Nordlys $\mathrm{F}+$ detector. Two different ion beam conditions, $30 \mathrm{kV}(300 \mathrm{pA})$ and $16 \mathrm{kV}(150 \mathrm{pA})$, were used to assess any differences in milling and EBSD performance. A $10 \mathrm{kV}, 4 \mathrm{nA}$ electron beam was used for acquiring the EBSD data in both cases. Relatively small regions were analyzed to minimize drift.

Initial results exhibited reasonable indexing in both phases, as shown in FIGs 2-3. Indexing at grain boundaries was poor, but grains $\sim 100 \mathrm{~nm}$ in size were detected accurately. No significant data quality differences from the two ion beam conditions were observed. Ongoing work is focused on exploring additional beam parameters and reconstruction methods to improve data quality.

\section{REFERENCES}

[1] G.G.E. Seward, et al. "In situ SEM-EBSD observations of the hcp to bcc phase transformation in commercially pure titanium" Acta Materialia 52 (2004) pp. 821-832 .

[2] M.D. Uchic. (2011). Serial Sectioning Methods for Generating 3D Characterization Data of Grain and Precipitate-Scale Microstructures. In: S. Gosh, D. Dimiduk. Computational Methods for Microstructure-Property Relationships. NY: Springer. pp. 31-52.

[3] R. DiDomizio et al. "Evaluation of a Ni-20-Cr alloy processed by multi-axis forging" Materials Science Forum. 503-504 (2006) pp. 793-798.

[4] L. Giannuzzi, F. Stevie (Eds.) (2005). Introduction to Focused Ion Beams. NY: Springer. 


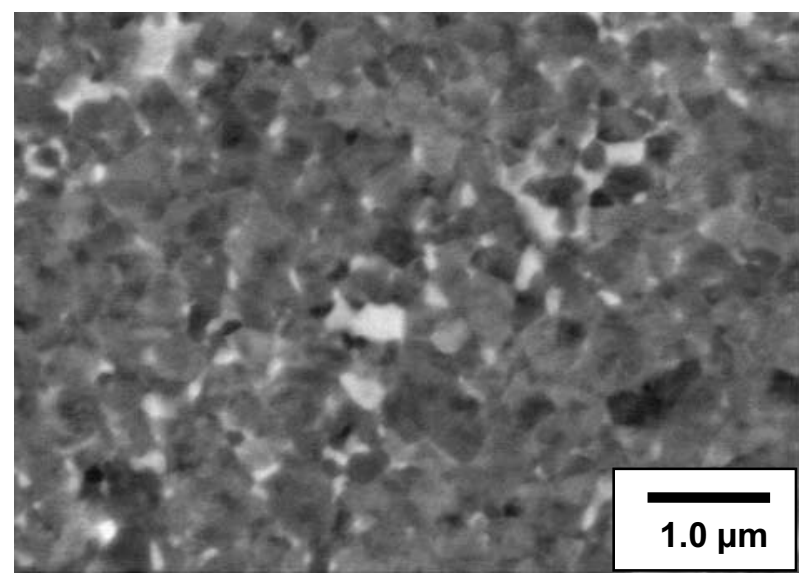

FIG. 1. BSE image of the Ti alloy. Bright regions $=$ beta phase. Grey regions $=$ alpha phase . Note that the image is from a different area than FIG 2, but representative of the microstructure.

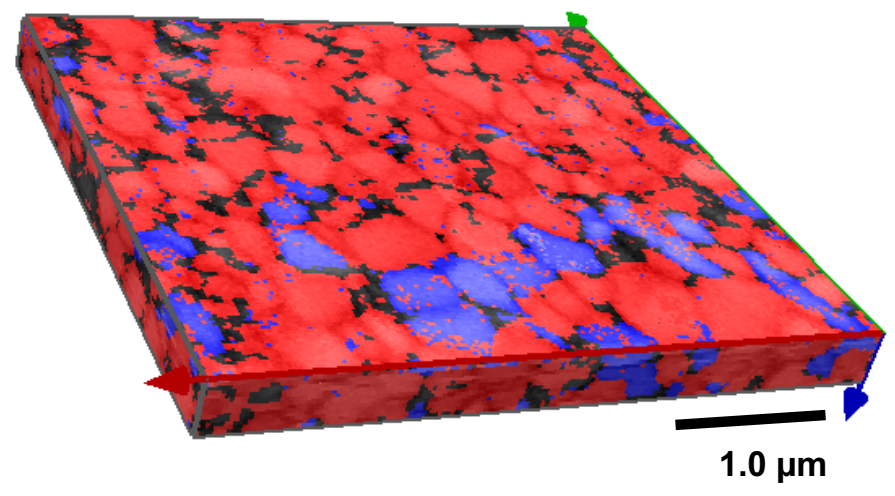

FIG. 2. Two-phase indexing in the $30 \mathrm{kV}$ ion beam data set. Phase coloring over band contrast map reconstruction. $\mathrm{RED}=$ alpha phase. BLUE = beta phase. Aggressive noise reduction applied to fill grain boundaries.
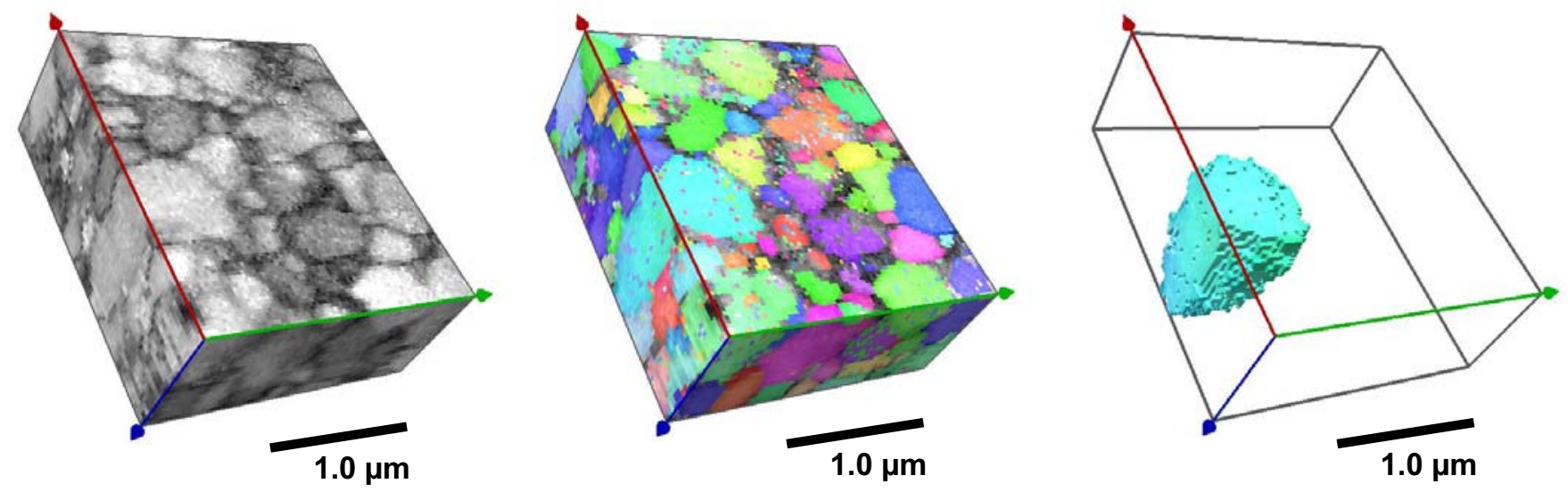

FIG. 3. Cropped region from $16 \mathrm{kV}$ ion beam data set. LEFT $=$ band contrast map reconstruction. MIDDLE $=$ IPF X coloring over band contrast (red is X-direction). RIGHT = sample alpha grain extracted from reconstructed volume. Aggressive noise reduction applied to fill grain boundaries.

The portions of this work completed at GE Global Research were performed under award $70 N A N B 7 H 7038$ from National Institute of Standards \& Technology (NIST), U.S. Department of Commerce. The statements, findings, conclusions, and recommendations are those of the authors and do not necessarily reflect the views of the NIST or the U.S. Department of Commerce. 